constant is not much sounder than a similar assumption for the Green's function. However, the fact that the partial $\mathbf{S}$ matrix here obtained is unitary makes us feel that this covariant calculation presents some progress over the Born approximation applied to this term.

\section{APPENDIX}

In this section we evaluate the integral appearing in Eq. (9),

$$
\begin{array}{r}
\delta M=\frac{3 g^{2}}{16 \pi^{2}} \int_{0}^{1} d u\{[(u-1) \gamma P-m] \\
\quad \times \ln \left[\frac{(1-u) \mu^{2}+u m^{2}+u(1-u) P^{2}}{(1-u) \mu^{2}+u^{2} m^{2}}\right] \\
\left.+(\gamma P+m) \frac{2 m^{2} u^{2}(1-u)}{m^{2} u^{2}+(1-u) \mu^{2}}\right\} .
\end{array}
$$

The integration is carried out in the reference frame in which the spatial components of $P$ are zero and the dimensionless quantities $\alpha=\mu / m$ and $\epsilon=P_{0} / m$ are introduced. The real part of $\delta M$ is obtained by straightforward integration with absolute value signs placed on the argument of the logarithm:

$$
\begin{aligned}
& \operatorname{Re} \delta M=\left(3 g^{2} / 16 \pi^{2}\right) m\left\{-1+2 \alpha^{2}+\left(1+3 \alpha^{2}-2 \alpha^{4}\right.\right. \\
& \left.+\left(\alpha^{2}-1\right) / \epsilon^{2}\right) \ln \alpha+\alpha^{3}\left(2 \alpha^{2}-7\right)\left(4-\alpha^{2}\right)^{-\frac{1}{2}} \\
& \times \tan ^{-1} \alpha\left(4-\alpha^{2}\right)^{\frac{1}{2}}-\frac{1}{2} B(\epsilon) \ln F(\epsilon) \\
& +\frac{1}{2} \gamma_{0} \epsilon\left[1-3 \alpha^{2}+\left(1-\alpha^{2}\right) / \epsilon^{2}-\left(1+4 \alpha^{2}\right.\right. \\
& \left.-3 \alpha^{4}+2 \alpha^{2} / \epsilon^{2}-\left(1-\alpha^{2}\right)^{2} / \epsilon^{4}\right) \ln \alpha \\
& -\alpha^{3}\left(3 \alpha^{2}-10\right)\left(4-\alpha^{2}\right)^{-\frac{1}{2}} \tan ^{-1} \alpha\left(4-\alpha^{2}\right)^{-\frac{1}{2}} \\
& \left.\left.+\left(1-\left(\alpha^{2}-1\right) / \epsilon^{2}\right) \frac{1}{2} B(\epsilon) \ln F(\epsilon)\right]\right\} \text {. } \\
& B(\epsilon)=\left(1-2\left(1+\alpha^{2}\right) / \epsilon^{2}+\left(1-\alpha^{2}\right)^{2} / \epsilon^{4}\right)^{\frac{1}{2}}
\end{aligned}
$$

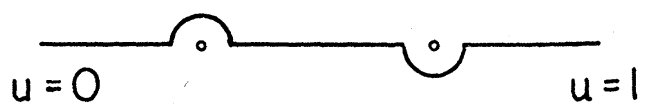

FIG. 2. Path of integration for the variable $u$.

and

$$
F(\epsilon)=\frac{1-\left(1+\alpha^{2}\right) / \epsilon^{2}-B(\epsilon)}{1-\left(1+\alpha^{2}\right) / \epsilon^{2}+B(\epsilon)} .
$$

To obtain the imaginary part of $\delta M$, we note that the polynomial $\alpha^{2}(1-u)+u-\epsilon^{2} u(1-u)$ which appears in the numerator of the logarithm in Eq. (9) has two roots in the region of integration $0 \leqslant u \leqslant 1$,

$$
u=\left(\epsilon^{2}-1+\alpha^{2}\right) / 2 \epsilon^{2} \pm \frac{1}{2} B(\epsilon) .
$$

and that it is negative between these roots. The values of the logarithm appropriate to the three segments of the interval may be inferred from the path of integration shown in Fig. 2, which follows from the choice of Green's functions for outgoing waves. The imaginary part of the mass operator is therefore

$$
\begin{aligned}
\operatorname{Im} \delta M=-( & \left(3 g^{2} / 16 \pi\right) m \\
& \times\left\{-1+\frac{1}{2} \gamma_{0} \epsilon\left[1+\left(1-\alpha^{2}\right) / \epsilon\right]\right\} B(\epsilon) .
\end{aligned}
$$

The numerical value of $\alpha^{-1}=M / \mu=6.70$ may be used to simplify the expressions in Eqs. (A.1) and (A.3) to

$$
\begin{gathered}
\delta M=\frac{3 g^{2} m}{16 \pi^{2}}\left\{-2.998+1.862 / \epsilon^{2}-\frac{1}{2} B(\epsilon) \ln F(\epsilon)\right. \\
+\gamma_{0} \epsilon\left[1.504+0.531 / \epsilon^{2}-0.910 / \epsilon^{4}\right. \\
\left.+\frac{1}{4}\left(1+0.9778 / \epsilon^{2}\right) B(\epsilon) \ln F(\epsilon)\right] \\
\left.+i \pi\left[-1+\frac{1}{2} \gamma_{0} \epsilon\left(1+0.9778 / \epsilon^{2}\right)\right] B(\epsilon)\right\} .
\end{gathered}
$$

\title{
A Covariant Meson-Nucleon Equation
}

\author{
Stanley Deser* and Paul C. Martin* \\ Harvard University, Cambridge, Massachusetts
}

(Received March 9, 1953)

\begin{abstract}
A relativistic meson-nucleon two-body equation is derived in a form suitable for carrying out renormalization. Methods for determining the interaction kernel and classifying its terms are discussed. A reduction of the equation to three dimensions is carried out and the approximations involved in this procedure are examined. The resulting equation agrees with a corresponding one derived by Tamm-Dancoff methods.
\end{abstract}

\section{INTRODUCTION}

GEVERAL investigations ${ }^{1}$ have been undertaken $\boldsymbol{N}$ recently with the aim of improving the Born approximation results for pion-nucleon scattering. It is the purpose of this note to point out that such a boson-

* National Science Foundation Predoctoral Fellow.

1 Dyson, Schweber, and Visscher, Phys. Rev. 90, 372 (1953). fermion system may be described to advantage by means of a covariant two-body equation akin to the one employed in the two-nucleon problem. ${ }^{2-4} \mathrm{We}$ shall derive such an equation for the pion-nucleon Green's

${ }^{2}$ J. Schwinger, Proc. Natl. Acad. Sci. U.S. 37, 452 (1951); also unpublished lectures at Harvard.

${ }^{3}$ H. A. Bethe and E. E. Salpeter, Phys. Rev. 84, 1232 (1951).

${ }^{4}$ M. Gell-Mann and F. Low, Phys. Rev. 84, 350 (1951). 
1076

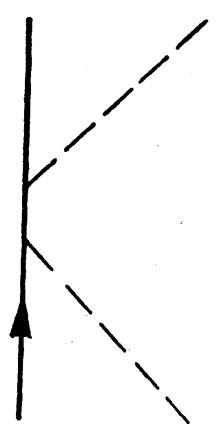

(a)

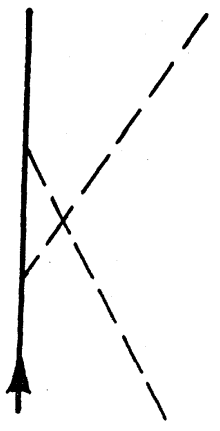

(b)
Fig. 1. The lowest order "Compton" interactions.

function, the latter being defined in a manner analogous to the two-nucleon Green's function. A symbolic equation for the interaction kernel of this problem yields on iteration all the irreducible ${ }^{3}$ terms of the kernel to any desired order. Approximations to the mesonnucleon (M.N.) equation may be based on approximations to this interaction kernel. For a given kernel, the M.N. equation may be reduced to three-dimensional form ${ }^{5-7}$ with the aid of certain assumptions. In particular, it is possible to reduce the equation for the M.N. Green's function with the interaction corresponding to the two simplest "Compton" terms (Fig. 1). The equation obtained is the same as one previously derived using a Fock space representation. ${ }^{1}$

\section{DERIVATION OF FOUR-DIMENSIONAL EQUATION}

We shall employ the notation and results of Schwinger ${ }^{2}$ as modified for pseudoscalar meson theory, ${ }^{8}$ but including the isotopic spin matrix in the $\gamma_{5}(\xi)$ or $\Gamma_{5}(\xi)$ vertex operators. The M.N. Green's function is defined by

$$
\begin{aligned}
G_{\mathrm{MN}}\left(x x^{\prime}, \xi_{1} \xi_{2}\right) & =\left(x \xi_{1}\left|G_{\mathrm{MN}}\right| x^{\prime} \xi_{2}\right) \\
= & -\left\langle\left(\psi(x) \bar{\psi}\left(x^{\prime}\right) \phi\left(\xi_{1}\right) \phi\left(\xi_{2}\right)\right)_{+}\right\rangle \epsilon\left(x-x^{\prime}\right) .
\end{aligned}
$$

The desired form for the M.N. equation is

$$
\begin{aligned}
& \left(F E-I_{\mathrm{MN}}\right) G_{\mathrm{MN}} \\
& \quad=\left[(\gamma p+M)\left(k^{2}+\mu^{2}+\Pi\right)-I_{\mathrm{MN}}\right] G_{\mathrm{MN}}=1,
\end{aligned}
$$

where $M$ is the nucleon mass operator and $\Pi$ the meson polarization operator. It is apparent from this equation that renormalization of the separate particle propagation functions may be carried out in the usual manner; this leaves only the finite parts of the $M$ and $\Pi$ operators which we shall neglect hereafter. The extra renormalization needed in pseudoscalar theory, that of direct mesonmeson scattering, is to be included in the renormalization of $\Delta_{+}$and offers no special difficulty. This renor-

5 E. E. Salpeter, Phys. Rev. 87, 328 (1952).

${ }^{6}$ M. M. Levy, Phys. Rev. 88, 72, 725 (1952).

${ }^{7}$ A. Klein, Phys. Rev. 90, 1090 (1953).

${ }^{8}$ S. F. Edwards, Phys. Rev. 90, 284 (1953). malization, like those of $\Gamma_{5}$ and $G_{+}$, must be carried out when these terms occur in $I_{\mathrm{MN}}$. A further complication arises due to the dual role of the meson as particle and virtual field quantum; that is, after $I_{\mathrm{MN}}$ has been renormalized, its iterates may still diverge. In that case, the divergences arising in the "ladder" process must be renormalized as well. It is only with such a prescription that the meson-nucleon equation yields finite solutions.

Two alternative methods may be used to determine $I_{\mathrm{MN}}$. The first employs the fact that given the Green's function for one nucleon in an external meson field, it is possible to determine the one-nucleon $n$-meson Green's function; ${ }^{9}$ the second method is closer to that employed in the two nucleon problem and treats the particles on a more equal footing.

In the first method, we note that the M.N. Green's function is related to that of one nucleon in an external meson field by

$$
\begin{aligned}
G_{\mathrm{MN}}\left(x x^{\prime}, \xi_{1} \xi_{2}\right)=\Delta_{+}\left(\xi_{1}, \xi_{2}\right) G_{+} & \left(x, x^{\prime}\right) \\
& +\left.\frac{1}{i \delta K\left(\xi_{1}\right) \delta K\left(\xi_{2}\right)}\right|_{K=0}
\end{aligned}
$$

where $K$ is the external meson source, set equal to zero after differentiation. This follows immediately from Eq. (1) and the identities

$$
\begin{aligned}
& \begin{array}{l}
\left.\frac{1}{i} \frac{\delta^{2} G_{+}\left(x, x^{\prime}\right)}{\delta K\left(\xi_{1}\right) \delta K\left(\xi_{2}\right)}\right|_{K=0} \\
=-\left\langle\left(\psi(x) \bar{\psi}\left(x^{\prime}\right) \phi\left(\xi_{1}\right) \phi\left(\xi_{2}\right)\right)_{+}\right\rangle \epsilon\left(x-x^{\prime}\right)
\end{array} \\
& \quad+\left\langle\left(\psi(x) \bar{\psi}\left(x^{\prime}\right)\right)_{+}\right\rangle \epsilon\left(x-x^{\prime}\right)\left\langle\left(\phi\left(\xi_{1}\right) \phi\left(\xi_{2}\right)\right)_{+}\right\rangle
\end{aligned}
$$

The variational derivative may be evaluated symbolically:

$$
\begin{aligned}
& \left.\frac{\delta^{2} G_{+}}{\delta K\left(\xi_{1}\right) \delta K\left(\xi_{2}\right)}\right|_{K=0} \\
& =g^{2}\left[G_{+} \Delta_{+}\left(\xi_{1}, \xi^{\prime}\right) \Gamma_{5}\left(\xi^{\prime}\right) G_{+} \Gamma_{5}\left(\xi^{\prime \prime}\right) \Delta_{+}\left(\xi^{\prime \prime}, \xi_{2}\right) G_{+}\right. \\
& \quad+G_{+} \Delta_{+}\left(\xi_{2}, \xi^{\prime}\right) \Gamma_{5}\left(\xi^{\prime}\right) G_{+} \Gamma_{5}\left(\xi^{\prime \prime}\right) \Delta_{+}\left(\xi^{\prime \prime}, \xi_{1}\right) G_{+} \\
& \quad+G_{+} \frac{\delta \Gamma_{5}\left(\xi^{\prime}\right)}{\delta g K\left(\xi_{2}\right)} \Delta_{+}\left(\xi^{\prime}, \xi_{1}\right) G_{+} \\
& \left.+G_{+} \Gamma_{5}\left(\xi^{\prime}\right) \frac{\delta \Delta_{+}\left(\xi^{\prime}, \xi_{1}\right)}{\delta g K\left(\xi_{2}\right)} G_{+}\right]_{K=0}
\end{aligned}
$$

Matrix notation has been employed in the above for only the nucleon coordinates, and a summation con-

${ }^{9}$ The possibility of treating Compton scattering by use of variational derivatives of the one fermion Green's function has also been noted by R. Utiyama et al., Progr. Theoret. Phys. (Japan) 8, 77 (1952). 
vention has been introduced for the meson coordinates including isotopic spin indices. The last term in Eq. (6) may be seen to vanish since $\left\langle(\phi \phi \phi)_{+}\right\rangle_{K=0}$ and $\langle\phi\rangle_{K=0}$ are zero. In the former case this is on account of a generalized Furry theorem for pseudoscalar symmetric theory. Thus $G_{\mathrm{MN}}$ may be obtained formally in closed form in terms of $G_{+}, \Delta_{+}, \Gamma_{5}$, and $\delta \Gamma_{5} / \delta K$; unfortunately, these quantities themselves are known only in series expansion. $I_{\mathrm{MN}}$ also may be obtained in closed form by substituting Eq. (6) into Eq. (2). Here again, only expansion is practicable, but a given approximation to $I_{\mathrm{MN}}$ may be inserted into Eq. (2) to yield a solution for $G_{\mathrm{MN}}$ which is not a power series approximation to $G_{\mathrm{MN}}$.

In the second method, the equation for $I_{\mathrm{MN}}$ is recast into a form more in correspondence with the two nucleon problem. From the definition of $G_{M N}$ and the equation of motion for the operator $\psi$, it will be seen that

$\mathfrak{F} G_{\mathrm{MN}}=\left(\gamma p+M-g \gamma_{5}\langle\phi\rangle+i g \gamma_{5} \delta / \delta K\right) G_{\mathrm{MN}}$

and that therefore

$$
=\Delta_{+}+\langle\phi\rangle\langle\phi\rangle \text {, }
$$

$$
E \mathfrak{F} G_{\mathrm{MN}}=1+E\langle\phi\rangle\langle\phi\rangle .
$$

Comparing Eq. (8) with the desired form, Eq. (2), and using $\delta G_{\mathrm{MN}} / \delta K$ as obtained by differentiating Eq. (2), we find a symbolic equation for $I_{\mathrm{MN}}$ of the form

$$
\begin{aligned}
& \left(I_{\mathrm{MN}} G_{\mathrm{MN}}\right)\left(\xi_{1}, \xi_{2}\right) \\
& =-i g \gamma_{5}(\xi) G_{+} \frac{\delta}{\delta K(\xi)}\left(I_{\mathrm{MN}} G_{\mathrm{MN}}\right)\left(\xi_{1}, \xi_{2}\right) \\
& +i g \gamma_{5}(\xi) \frac{\delta E\left(\xi_{1}, \xi^{\prime}\right)}{\delta K(\xi)} G_{\mathrm{MN}}\left(\xi^{\prime}, \xi_{2}\right)+E\left(\xi_{1}, \xi\right)\langle\phi(\xi)\rangle\left\langle\phi\left(\xi_{2}\right)\right\rangle
\end{aligned}
$$

This equation may be iterated to obtain $I_{\mathrm{MN}} G_{\mathrm{MN}}$ to any desired order by substituting the last two terms of the right-hand side into $-i g \gamma_{5}(\xi) G_{+}(\delta / \delta K(\xi)) I_{\mathrm{MN}} G_{\mathrm{MN}}$. Indefinite repetition of this procedure generates two distinct groups of terms: the first series, arising from $E\langle\phi\rangle\langle\phi\rangle$ consists of generalized "Compton" terms, in which at least one of the real mesons interacts directly with the nucleon; the other series, typified by Fig. 2, contains "indirect" types of interaction in which the real mesons and nucleon affect each other only through the nucleon's virtual meson cloud. This latter sequence results from the middle term on the right hand side of Eq. (9). Had we derived the interaction using Eq. (6) we would have found this series of processes along with some of those of the first series included in the expansion of $\delta \Gamma / \delta K$.

It is easy to verify that the lowest order nonvanishing terms in the Compton and indirect series are, respectively, ${ }^{10}$

$$
I_{C}{ }^{(0)}\left(\xi, \xi^{\prime}\right)=-i g^{2}\left[\gamma_{5}(\xi) G_{+}{ }^{(0)} \gamma_{5}\left(\xi^{\prime}\right)+\gamma_{5}\left(\xi^{\prime}\right) G_{+}{ }^{(0)} \gamma_{5}(\xi)\right]
$$

${ }^{10} \mathrm{~A}$ superscript zero is used to denote the propagation function of a noninteracting field. (or explicitly,

$$
\begin{aligned}
& I_{C}{ }^{(0)}\left(x, x^{\prime} ; \xi_{i}, \xi_{j}{ }^{\prime}\right) \\
& =-i g^{2}\left[\gamma_{5} \tau_{i} \delta(x-\xi) G_{+}{ }^{(0)}\left(x, x^{\prime}\right) \gamma_{5} \tau_{j} \delta\left(x^{\prime}-\xi^{\prime}\right)\right. \\
& \left.+\gamma_{5} \tau_{j} \delta\left(x-\xi^{\prime}\right) G_{+}{ }^{(0)}\left(x, x^{\prime}\right) \gamma_{5} \tau_{i} \delta\left(x^{\prime}-\xi\right)\right] \text {, } \\
& \text { and } \\
& \times \operatorname{Tr}\left[\gamma_{5}\left(\xi_{1}\right) G_{+}{ }^{(0)} \gamma_{5}\left(\eta^{\prime}\right) G_{+}{ }^{(0)} \gamma_{5}(\eta) G_{+}{ }^{(0)} \gamma_{5}\left(\xi_{2}\right) G_{+}{ }^{(0)}\right. \\
& +\gamma_{5}\left(\xi_{1}\right) G_{+}{ }^{(0)} \gamma_{5}(\eta) G_{+}{ }^{(0)} \gamma_{5}\left(\xi_{2}\right) G_{+}{ }^{(0)} \gamma_{5}\left(\eta^{\prime}\right) G_{+}{ }^{(0)} \\
& \left.+\gamma_{5}\left(\xi_{1}\right) G_{+}{ }^{(0)} \gamma_{5}(\eta) G_{+}{ }^{(0)} \gamma_{5}\left(\eta^{\prime}\right) G_{+}{ }^{(0)} \gamma_{5}\left(\xi_{2}\right) G_{+}{ }^{(0)}\right] \\
& + \text { terms with } \xi_{1} \text { and } \xi_{2} \text { interchanged. }
\end{aligned}
$$

- As in the two-nucleon problem, ${ }^{4}$ only irreducible terms contribute to $I_{\mathrm{MN}}$. Since radiative corrections to the nucleon propagation function and to $\gamma_{5}\left(\xi^{\prime}\right)$ yield reducible graphs, the terms from Fig. 1(a) will take the form $\gamma_{5}(\xi) G_{+}{ }^{(0)} \gamma_{5}\left(\xi^{\prime}\right)$ in higher order approximations.

\section{REDUCTION TO THREE DIMENSIONS}

By adaptation of now standard techniques, ${ }^{5-7}$ the four-dimensional Eq. (2) may be reduced to threedimensional form with an interaction operator correct to any order. Equation (2) may be written in momentum space in terms of the total four-momentum $P$ and one-half the difference of the nucleon and meson momenta, $p$,

$$
\begin{aligned}
& \left(\gamma\left(\frac{1}{2} P+p\right)+M\right)\left(\left(\frac{1}{2} P-p\right)^{2}+\mu^{2}\right) G_{\mathrm{MN}}\left(p, p^{\prime} ; P\right) \\
& \times \int I_{\mathrm{MN}}\left(p, p^{\prime \prime} ; P\right) d p^{\prime \prime} G_{\mathrm{MN}}\left(p^{\prime \prime}, p^{\prime} ; P\right)+\delta\left(p-p^{\prime}\right) .
\end{aligned}
$$

The first step in the derivation employs the adiabatic approximation; we assume that the three-dimensional Green's function,

$$
\begin{aligned}
G\left(\mathbf{p}, \mathbf{p}^{\prime} ; P\right)=\frac{1}{(2 \pi)^{\frac{1}{2}}} & \int d p_{0} G\left(p, \mathbf{p}^{\prime} ; P\right) \\
& =\frac{1}{2 \pi} \int d p_{0} d p_{0}{ }^{\prime} G_{\mathrm{MN}}\left(p, p^{\prime} ; P\right),
\end{aligned}
$$

is related to $G\left(p, \mathbf{p}^{\prime} ; P\right)$ by

$$
\begin{aligned}
G\left(p, \mathbf{p}^{\prime} ; P\right)= & (2 \pi)^{-\frac{1}{2}} i \Omega(p, P) 2 \omega_{\mathrm{p}}\left[\left(P_{0}-E_{\mathrm{p}}-\omega_{\mathrm{p}}\right) \Lambda_{+}(\mathbf{p})\right. \\
& \left.+\left(P_{0}+E_{\mathrm{p}}+\omega_{\mathrm{p}}\right) \Lambda_{-}(\mathbf{p})\right] G\left(\mathbf{p}, \mathbf{p}^{\prime} ; P\right)
\end{aligned}
$$

where, in the reference frame in which $\mathbf{P}=0$,

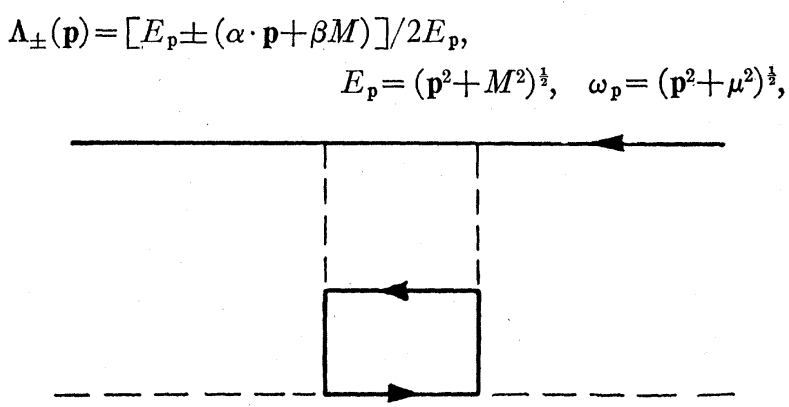

FIG. 2. A typical lowest order indirect interaction. 
and

$$
\begin{aligned}
& \Omega(p, P)=\left[\alpha \cdot \mathbf{p}+\beta M-\left(\frac{1}{2} P_{0}+p_{0}\right)\right]^{-1}\left[\omega_{\mathrm{p}}{ }^{2}-\left(\frac{1}{2} P_{0}-p_{0}\right)^{2}\right]^{-1} \\
&= {\left[\frac{\Lambda_{+}(\mathbf{p})}{E_{\mathrm{p}}-\frac{1}{2} P_{0}-p_{0}}-\frac{\Lambda_{-}(\mathbf{p})}{E_{\mathrm{p}}+\frac{1}{2} P_{0}+p_{0}}\right] } \\
& \times \frac{1}{2 \omega_{\mathrm{p}}}\left[\frac{1}{\omega_{\mathrm{p}}-\frac{1}{2} P_{0}+p_{0}}+\frac{1}{\omega_{\mathrm{p}}+\frac{1}{2} P_{0}-p_{0}}\right]
\end{aligned}
$$

Inserting Eq. (14) into Eq. (12), we obtain

$$
\begin{aligned}
G\left(\mathbf{p}, \mathbf{p}^{\prime} ; P_{0}\right)= & (2 \pi i)^{-1} \int \Omega\left(p, P_{0}\right) d p_{0} \gamma_{0} I\left(p, p^{\prime \prime} ; P_{0}\right) \gamma_{0} d p^{\prime \prime} \\
& \times \Omega\left(p^{\prime \prime}, P_{0}\right) G\left(p^{\prime \prime}, \mathbf{p}^{\prime} ; P_{0}\right) \\
& +(2 \pi)^{-1} \int d p_{0} \Omega\left(p, P_{0}\right) \gamma_{0} \delta\left(\mathbf{p}-\mathbf{p}^{\prime}\right)
\end{aligned}
$$

as the equation for the Green's function. ${ }^{11}$ In scattering problems, the quantities of interests are matrix elements of $G$ between free particle states at times prior and subsequent to the interaction. At these times, we require that one nucleon and one meson be present, the meson having positive frequencies. This means that we need only be concerned with that part of the Green's function which has nonvanishing matrix elements between such states, and desire an equation for this part alone. We denote it by ${ }_{+} G_{+}$where the plus sign on either side indicates the restriction of $G$ to those matrix elements on that side which satisfy the above criteria. In other words, ${ }_{+} G_{+}=\Lambda_{+}(\mathbf{p}) \omega_{+}(\mathbf{p}) G\left(\mathbf{p}, \mathbf{p}^{\prime} ; P\right) \Lambda_{+}\left(\mathbf{p}^{\prime}\right) \omega_{+}\left(\mathbf{p}^{\prime}\right)$, where the $\omega_{+}$operators select that part of $G_{\mathrm{MN}}$ which contains the proper meson frequencies. With these restrictions, Eq. (16) becomes

$$
\begin{aligned}
& { }_{+} G_{+}\left(\mathbf{p}, \mathbf{p}^{\prime} ; P_{0}\right)=(2 \pi i)^{-1} \int\left(E_{\mathrm{p}}-\frac{1}{2} P_{0}-p_{0}\right)^{-1} \\
& \times \Lambda_{+}(\mathbf{p})\left(2 \omega_{\mathrm{p}}\right)^{-1}\left(\omega_{\mathrm{p}}-\frac{1}{2} P_{0}+p_{0}\right)^{-1} d p_{0} \\
& \times \gamma_{0} I\left(p, p^{\prime \prime} ; P_{0}\right) \gamma_{0} d p^{\prime \prime} \Omega\left(p^{\prime \prime}, P_{0}\right) G_{+}\left(p^{\prime \prime}, \mathbf{p}^{\prime} ; P_{0}\right) \\
& +\left(2 \omega_{\mathrm{p}} i\right)^{-1} \Lambda_{+}(\mathbf{p}) \gamma_{0} \delta\left(\mathbf{p}-\mathbf{p}^{\prime}\right) \Lambda_{+}\left(\mathbf{p}^{\prime}\right) .
\end{aligned}
$$

It is clear that this argument cannot be used to eliminate the factors

and

$$
\begin{gathered}
\left(E_{\mathrm{p}^{\prime \prime}}+\frac{1}{2} P_{0}+p_{0}{ }^{\prime \prime}\right)^{-1} \Lambda_{-}\left(\mathbf{p}^{\prime \prime}\right) \\
\left(\omega_{\mathrm{p}^{\prime \prime}}+\frac{1}{2} P_{0}-p_{0}{ }^{\prime \prime}\right)^{-1}
\end{gathered}
$$

occurring in $\Omega\left(p^{\prime \prime}\right)$ on the right-hand side of Eq. (17). However as long as we have $G_{+}$and not ${ }_{+} G_{+}$on the right-hand side of $\mathrm{Eq}$. (17), the reduction made in going from Eq. (16) to Eq. (17) does not suffice for our purpose. The simplest assumption, rigorous only when there is no interaction, is to replace $G_{+}$by ${ }_{+} G_{+}$, or equivalently, to replace $I_{+}$by ${ }_{+} I_{+}$. The portions of $I$

\footnotetext{
${ }^{11}$ In contrast to the result in the two-nucleon problem, this equation is an equation for $G$ and not for certain Dirac spinor components of $G$.
}

that are thereby excluded may be reinserted to any desired extent through the use of more complex effective interaction operators (which are reducible in a fourdimensional sense). These are obtained by iteration processes described elsewhere and are of higher order than the covariant kernel from which they arise; hence this procedure has been carried out ${ }^{6,7}$ together with the inclusion of irreducible interaction terms of comparable order in $g^{2}$. Clearly, at any stage of the three-dimensional procedure, only part of the initial kernel will be included so that the three-dimensional effective interaction of a given order will in some sense contain less than the four-dimensional kernel to that order. This is not to say that there is mathematical justification for preferring the latter, since for neither approximation is the error involved known.

Since it is not our aim to solve Eq. (17) in any approximation, we shall not dwell on the more involved aspects of this procedure. We merely illustrate it by reduction of Eq. (12) with the lowest order interaction, Eq. (10), and with the approximation $I_{+} \rightarrow_{+} I_{+} \cdot{ }^{12}$ In the coordinate system of Eq. (12), this interaction is

$$
\begin{aligned}
& I_{C}{ }^{(0)}\left(p, p^{\prime} ; P_{0}\right)= \\
& \quad-\frac{i g^{2}}{(2 \pi)^{4}} \gamma_{5}\left[\frac{\tau \tau^{\prime}}{-\gamma_{0} P_{0}+M}+\frac{\tau^{\prime} \tau}{\gamma\left(p+p^{\prime}\right)+M}\right] \gamma_{5} .
\end{aligned}
$$

Insertion of Eq. (18) into the ${ }_{+} I_{+}$version of Eq. (17) and integration over the relative energy variables yields

$$
\begin{aligned}
\left(P_{0}-E_{\mathbf{p}}-\omega_{\mathrm{p}}\right) G_{i k}\left(\mathbf{p}, \mathbf{p}^{\prime} ; P_{0}\right) & \\
= & \frac{-g^{2}}{(2 \pi)^{3}} \int\left[\frac{\left(1-\gamma_{0}\right) \tau_{i} \tau_{j}}{2\left(P_{0}-M\right)}+\frac{\left(1+\gamma_{0}\right) \tau_{i} \tau_{j}}{2\left(P_{0}+M\right)}\right. \\
& \left.+\frac{\Lambda_{-}\left(\mathbf{p}+\mathbf{p}^{\prime \prime}\right) \tau_{j} \tau_{i}}{P_{0}-E_{\mathbf{p}+\mathbf{p}^{\prime \prime}}-\omega_{\mathrm{p}}-\omega_{\mathbf{p}^{\prime \prime}}}+\frac{\Lambda_{+}\left(\mathbf{p}+\mathbf{p}^{\prime \prime}\right) \tau_{j} \tau_{i}}{P_{0}-E_{\mathrm{p}+\mathbf{p}^{\prime \prime}}-E_{\mathrm{p}}-E_{\mathbf{p}^{\prime \prime}}}\right] \\
& \quad \times \frac{d \mathbf{p}^{\prime \prime}}{2 \omega_{\mathrm{p}}} G_{j k}\left(\mathbf{p}^{\prime \prime}, \mathbf{p}^{\prime} ; P_{0}\right)+\left(2 \omega_{\mathrm{p}} i\right)^{-1} \delta\left(\mathbf{p}-\mathbf{p}^{\prime}\right) \delta_{i k} .
\end{aligned}
$$

In wave function form, this equation is the same as that obtained by a Tamm-Dancoff procedure previously. "The "uncrossed" term is troublesome in this three-dimensional formulation on account of its contact nature; however, precisely for this reason, the M.N. equation containing only this term has been solved directly in four dimensions. ${ }^{13}$ For charge and spin $\frac{3}{2}$ states, only the "crossed" term contributes and the contact term may be dropped in the three-dimensional investigations.

We are grateful to Professor R. Karplus, Professor J. Schwinger, and Dr. A. Klein for illuminating conversations and constructive criticism.

12 This approximation implies that no more than three nucleons may be present at a given time and restricts the number of permitted meson states.

${ }^{13} \mathrm{Karplus}$, Kivelson, and Martin, following paper [Phys. Rev. 90, 1072 (1953)]. 